\title{
Optimal Maneuver for Multiple Aircraft Conflict Resolution: A Braid Point of VIEW ${ }^{1}$
}

\author{
Jianghai $\mathrm{Hu}$ \\ jianghai@eecs.berkeley.edu \\ Electrical Eng. \& Comp. Science \\ Univ. of California at Berkeley
}

\author{
Maria Prandini \\ prandini@ing.unibs.it \\ Electrical Eng. for Automation \\ University of Brescia, Italy
}

\author{
Shankar Sastry \\ sastry@eecs.berkeley.edu \\ Electrical Eng. \& Comp. Science \\ Univ. of California at Berkeley
}

\begin{abstract}
In this paper, we study conflict resolution for multiple aircraft encounter situations on a plane. First, the homotopy types of resolution maneuvers for $n$ aircraft encounters are classified according to their images in the joint spacetime coordinates, which are shown to bear a one-to-one correspondence with the well-known pure braid group $\mathbf{P B}_{n}$. Energy is then proposed as the cost function for choosing among all conflict-free maneuvers the optimal one. For two aircraft encounters, analytic expressions of the optimal resolution maneuvers are obtained, and for the multiple aircraft case, convex optimization technique is used to find the optimal two-legged resolution maneuver within each type. The introduced solution, however, becomes computationally intractable as the number of aircraft increases. The use of the probabilistic resolution algorithm in [1] as "random type chooser" is then suggested as a randomized solution to the combinatorial optimization problem. Finally, simulation results are presented for some typical encounters.
\end{abstract}

\section{Introduction}

The current Air Traffic Management System (ATMS) is characterized by a network-based architecture where with few exceptions aircraft are forced to fly along predefined jetways connecting navigation beacons, [2]. Supposedly this architecture can guarantee safety in the sense that the aircraft encounter geometries and the locations of the more critical areas in term of traffic are known a priori. As a matter of fact, based on such information, the airspace has been decomposed into sectors. Each sector is managed by an Air Traffic Controller (ATC), which ensures aircraft separation by issuing appropriate trajectory specifications to the pilots. On the other hand, in such a rigidly structured architecture pilots are not allowed to choose direct or wind favorable routes to their desired destinations to optimize travel times and fuel consumption. Moreover, the dramatically increasing demand for air travel observed in recent years is likely to cause a degradation of

\footnotetext{
${ }^{1}$ Research supported by NSF KDI 9873474 , by NASA grant NAG 2-1039, by DARPA under grant F33615-98-C-3614, and by Honeywell, Inc. on DARPA contract B09350186.
}

not only the current ATMS performances, but also its safety level due to the increased work load for human operators supported by outdated tools.

One of the proposed innovations in future ATMS is the realization of the free flight paradigm, where the totally centralized, ground-based decision making of the current ATMS is shifted to the air to individual aircraft, with the exception of critical areas such as those over airports. This should help to decrease travel times, unplanned delays and fuel consumption. However, a set of challenging issues also arise for the new ATMS regarding its hierarchical, decentralized and hybrid nature, [3]. Among them guarantee of safety is the most important one. Thus the development of a conflict avoidance system is essential for the new ATMS.

In the context of ATMS, safety is typically characterized in terms of conflicts, i.e., situations where two aircraft come closer than a minimal allowed distance. Conflict avoidance is typically decomposed into two separate procedures: conflict detection and conflict resolution. In the conflict detection phase, potential conflict situations are predicted based on the current aircraft positions, headings and flight plans. This information is then used in the resolution phase to re-plan the trajectories of the aircraft involved in the conflict.

In this paper, we study the conflict resolution problem for encounter situations involving multiple aircraft. Although for the two aircraft case there are many contributions in the literature both in deterministic and probabilistic settings, the treatments of multiple aircraft case are relatively rare and unsystematic. Among them, we mention $[4,5],[6]$, and [7], respectively based on potential field method, genetic algorithms and semidefinite programming. A survey of various approaches to conflict detection and resolution is given in [8].

This paper is organized as follows. In Section 2, a formal classification of conflict resolution maneuvers for multiple aircraft encounters is presented. In Section 3, the notion of energy of maneuvers is introduced and resolution maneuvers with minimal energy are studied. Various necessary conditions for optimality are derived and, as a side result, the analytical expressions of the 
optimal resolutions for two aircraft encounters are obtained. As the number of aircraft increases, it is difficult, if not impossible, to get such an analytical expression. By focusing on those resolution maneuvers specified by a set of waypoints, we are able to use convex optimization to solve an approximation of the twolegged optimal resolution problem for each resolution type. Finally, in Section 4, the problem of choosing from the vast number of resolution types is considered, and a computationally efficient randomized optimization algorithm is introduced.

\section{Classification of Resolution Maneuvers}

In this section, we introduce a classification of resolution maneuvers involving multiple aircraft into qualitatively different types. Roughly speaking, two resolution maneuvers are classified as qualitatively identical — or of the same type - if there exists a continuous conflictfree deformation of one to the other. Hence switching between qualitatively different maneuvers cannot be done smoothly without incurring situations of higher risk of conflict. As a result, distinguishing between maneuvers based on their type is useful, since it is preferable for aircraft involved in an encounter situation to negotiate a certain resolution type at an early stage, and stick to it throughout the encounter.

Consider $n$ aircraft (numbered from 1 to $n$ ) flying at the same altitude, where each aircraft, say $i$, flies from position $a_{i} \in \mathbf{R}^{2}$, at time $t_{0}$, to position $b_{i} \in \mathbf{R}^{2}$, at time $t_{f}$. Denote with $\mathbf{P}_{i}$ the space of all continuous maneuvers in $\mathbf{R}^{2}$ which start from $a_{i}$ at time $t_{0}$ and end at $b_{i}$ at time $t_{f}$, i.e., $\mathbf{P}_{i} \triangleq\left\{\alpha_{i} \in C\left(T_{h}, \mathbf{R}^{2}\right)\right.$ : $\left.\alpha_{i}\left(t_{0}\right)=a_{i}, \alpha_{i}\left(t_{f}\right)=b_{i}\right\}$, where $C\left(T_{h}, \mathbf{R}^{2}\right)$ denotes the set of all continuous maps from $T_{h} \triangleq\left[t_{0}, t_{f}\right]$ to $\mathbf{R}^{2}$. Set $\mathbf{P}=\prod_{i=1}^{n} \mathbf{P}_{i}$. Each element $\alpha \in \mathbf{P}$ is called a (joint) maneuver for the $n$ aircraft encounter, or an $n$-maneuver. The minimum separation over encounter $(M S E)$ for an $n$-maneuver $\alpha$ is defined as:

$$
\Delta(\alpha) \triangleq \min _{1 \leq i<j \leq n} \inf _{t \in T_{h}}\left\|\alpha_{i}(t)-\alpha_{j}(t)\right\| .
$$

The set of conflict-free maneuvers is then given by

$$
\mathbf{P}(R) \triangleq\{\alpha \in \mathbf{P}: \Delta(\alpha)>R\},
$$

where $R$ is the prescribed horizontal safe distance. We distinguish different classes of maneuvers in $\mathbf{P}(R)$ according to the following equivalence relation:

Definition 1 (R-Homotopy) Two maneuvers $\alpha$ and $\beta$ in $\mathbf{P}(R)$ are said to be $R$-homotopic if there exists $H=\left(H_{1}, \cdots, H_{n}\right)$, where $H_{i}:[0,1] \times T_{h} \rightarrow \mathbf{R}^{2}, i=$ $1, \cdots, n$, are continuous maps, such that

1. $H(0, \cdot)=\left(H_{1}(0, \cdot), \cdots, H_{n}(0, \cdot)\right)=\left(\alpha_{1}, \cdots, \alpha_{n}\right)$;

2. $H(1, \cdot)=\left(H_{1}(1, \cdot), \cdots, H_{n}(1, \cdot)\right)=\left(\beta_{1}, \cdots, \beta_{n}\right)$;

3. For any $s \in[0,1], H(s, \cdot) \in \mathbf{P}(R)$.
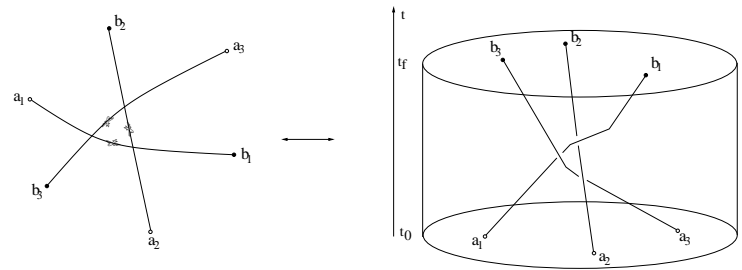

Figure 1: A 3-maneuver and its braid representation.

In other words, two maneuvers $\alpha$ and $\beta$ in $\mathbf{P}(R)$ are $R$-homotopic if there exists a continuous conflict-free deformation of $\alpha$ to $\beta$. It is easily verified that the $R$-homotopy relation is indeed an equivalence relation, hence it induces a partition of $\mathbf{P}(R)$ into equivalence classes. The objective of this section is to characterize the structure of these equivalence classes.

Set $\mathbf{a}=\left(a_{1}, \cdots, a_{n}\right)$ and $\mathbf{b}=\left(b_{1}, \cdots, b_{n}\right)$.

Definition 2 (Braids \& Pure Braids) An $n$-braid joining $\mathbf{a}$ to $\mathbf{b}$ is a set of $n$ non-intersecting curves $\left\{\gamma_{1}, \cdots, \gamma_{n}\right\}$ in $\mathbf{R}^{2} \times T_{h} \subset \mathbf{R}^{3}$ satisfying:

1. Each point $\left(a_{i}, t_{0}\right)$ is joined by exactly one curve to one of the points $\left(b_{j}, t_{f}\right)$ for some $j, 1 \leq j \leq n$;

2. The horizontal plane $t=t_{1}$ intersects each curve exactly once if $t_{1} \in\left[t_{0}, t_{f}\right]$ and never otherwise.

The set of all such braids is denoted with $\mathbf{B}(\mathbf{a}, \mathbf{b})$. If $i$ and $j$ are required to be identical in the first condition, the resulting braid is called pure. The set of all pure braids joining $\mathbf{a}$ to $\mathbf{b}$ is denoted with $\mathbf{P B}(\mathbf{a}, \mathbf{b})$.

There is a simple equivalence relation defined on $\mathbf{B}(\mathbf{a}, \mathbf{b})$, and hence on $\mathbf{P B}(\mathbf{a}, \mathbf{b})$ as well ([9]).

Definition 3 (String Isotopy) Two braids are said to be string isotopic if there exists a continuous deformation of one to the other satisfying all the conditions in Definition 2 throughout the deformation.

The reason we introduce the notion of braids is that there exists a very natural one-to-one correspondence between maneuvers in $\mathbf{P}(0)$ and pure braids in $\mathbf{P B}(\mathbf{a}, \mathbf{b})$. For each $n$-maneuver $\alpha=\left(\alpha_{1}, \cdots, \alpha_{n}\right) \in$ $\mathbf{P}(0)$, let $\gamma_{i}$ be the curve in $\mathbf{R}^{2} \times T_{h}$ determined by the image of map $t \mapsto\left(\alpha_{i}(t), t\right), t \in T_{h}$, which joins point $\left(a_{i}, t_{0}\right)$ to point $\left(b_{i}, t_{f}\right)$. Then, it is clear from the definition of $\mathbf{P}(0)$ that the set of $n$ curves $\left\{\gamma_{1}, \cdots, \gamma_{n}\right\}$ is indeed a pure braid in $\mathbf{P B}(\mathbf{a}, \mathbf{b})$, which we shall denote as $\hat{\alpha}$. Figure 1 shows a 3 -maneuver and its braid representation.

The map $\alpha \mapsto \hat{\alpha}$ can be verified to be a bijection between $\mathbf{P}(0)$ and $\mathbf{P B}(\mathbf{a}, \mathbf{b})$. Furthermore it is clear from the definitions that the following result holds.

Proposition 1 Two maneuvers $\alpha, \beta \in \mathbf{P}(0)$ are 0 homotopic if and only if $\hat{\alpha}$ and $\hat{\beta}$ are string isotopic. 
Therefore by Proposition 1, there is a one-to-one correspondence between the 0-homotopy classes of $\mathbf{P}(0)$ and the string isotopy classes of $\mathbf{P B}(\mathbf{a}, \mathbf{b})$.

A product operation on the set of all braids is defined as follows. For each $\hat{\alpha} \in \mathbf{B}(\mathbf{a}, \mathbf{b})$ and $\hat{\beta} \in \mathbf{B}(\mathbf{b}, \mathbf{c})$, the product $\hat{\gamma}=\hat{\alpha} \cdot \hat{\beta}$ is the braid in $\mathbf{B}(\mathbf{a}, \mathbf{c})$ obtained by concatenating the $n$ curves of $\hat{\beta}$ with those of $\hat{\alpha}$ and then renormalizing the $t$ axis such that the resultant $n$ curves connect $\left\{\left(a_{i}, t_{0}\right)\right\}_{i=1}^{n}$ to $\left\{\left(c_{i}, t_{f}\right)\right\}_{i=1}^{n}$. It can be checked that this product operation preserves string isotopy, hence it induces a product operation on the string isotopy classes of braids. Under this operation the isotopy classes of braids with the same starting and ending points, say $\mathbf{B}(\mathbf{a}, \mathbf{a})$, forms a group. We denote this group as $\mathbf{B}_{n}$. Similarly, the isotopy classes of pure braids $\mathbf{P B}(\mathbf{a}, \mathbf{a})$ forms under the same product operation a group, which we denote as $\mathbf{P B}_{n}$. The interested reader is referred to [1] and the references therein on braid theory for a detailed derivation of the above claims and the characterization of $\mathbf{P} \mathbf{B}_{n}$.

Now, if we fix a braid $\hat{\beta}$ in $\mathbf{P B}(\mathbf{b}, \mathbf{a})$, for each $\hat{\alpha} \in$ $\mathbf{P B}(\mathbf{a}, \mathbf{b}), \hat{\alpha} \cdot \hat{\beta}$ is a braid in $\mathbf{P B}(\mathbf{a}, \mathbf{a})$. It is easily verified that this map preserves string isotopy and actually maps the isotopy classes of $\mathbf{P B}(\mathbf{a}, \mathbf{b})$ bijectively to the isotopy classes of $\mathbf{P B}(\mathbf{a}, \mathbf{a})$, i.e., the elements of $\mathbf{P B}_{n}$. This fact combined with the result in Proposition 1 implies that there exists a bijection between the 0-homotopy classes of $\mathbf{P}(0)$ and the elements of $\mathbf{P} \mathbf{B}_{n}$.

A little thought reveals that the above considerations still hold for arbitrary $R$. Therefore,

Theorem 1 (Classification of $n$-Maneuvers) The $R$-homotopy classes of $n$-maneuvers in $\mathbf{P}(R)$ have a one-to-one correspondence with the elements of the group of pure $n$-braids $\mathbf{P B}_{n}$.

The group $\mathbf{P B}_{n}$ is described by a set of generators together with a set of relations defined on them. Therefore, Theorem 1 completely characterizes the structure of the homotopy types of resolution maneuvers for $n$ aircraft encounters. On the other hand, the description is unsatisfactory in practical terms, since in general the exact expression of $\mathbf{P} \mathbf{B}_{n}$ is very complicated. However, when $n$ is small, the result in Theorem 1 can have simple interpretations. Refer to [1] for further details.

\section{Optimal Resolution}

\subsection{Cost Function}

Two resolution maneuvers are of the same type if and only if there exists a continuous conflict-free deformation from one to the other. However, they may not be the same in terms of practical aspects such as travel distance, time delay, fuel consumption, etc.. Then, a question that naturally arises is: among all the representatives of a given type of maneuvers, which one is the "best"?

The answer to this question depends obviously on the evaluation criterion one chooses. Before we propose our cost function, let us re-define some notations. As in Section 2, we consider $n$ aircraft flying at the same altitude, with starting positions $a_{i} \in \mathbf{R}^{2}, i=1, \cdots, n$, at time $t_{0}$ and destination positions $b_{i} \in \mathbf{R}^{2}, i=1, \cdots, n$, at time $t_{f}$. In this section, the set of maneuvers for aircraft $i, \mathbf{P}_{i}$, is redefined to be the set of all continuous and piecewise smooth paths in $\mathbf{R}^{2}$ which start from $a_{i}$ at time $t_{0}$ and end at $b_{i}$ at time $t_{f} . \mathbf{P}(R)$ is redefined to be the set of all $n$-maneuvers with a MSE greater than or equal to $R$. This modification is made to ensure that $\mathbf{P}(R)$ is a closed set, so that for the optimization problems we encounter later, the optimal value is in fact attained by some maneuver in $\mathbf{P}(R)$.

Various cost functions can be proposed as performance index of a resolution maneuver. For example, given an $n$-maneuver $\alpha=\left(\alpha_{1}, \cdots, \alpha_{n}\right) \in \mathbf{P}$, one could consider the total length of the curves $\alpha_{1}, \cdots, \alpha_{n}$, with the length of $\alpha_{i}$ defined by: $L\left(\alpha_{i}\right)=\int_{t_{0}}^{t_{f}}\left\|\dot{\alpha}_{i}(t)\right\| d t$. However, since $L$ is a function only of the image of $\alpha_{i}$, a reparameterization of $\alpha_{i}$ yields a curve with the same cost. This is undesirable since in the context of air travel, maneuvers with less speed variation should be preferred. For this purpose, we propose as cost function the energy of an $n$-maneuver $\alpha$, which is defined as

$$
J(\alpha) \triangleq \sum_{i=1}^{n} J\left(\alpha_{i}\right),
$$

where $J\left(\alpha_{i}\right)=\int_{t_{0}}^{t_{f}}\left\|\dot{\alpha}_{i}(t)\right\|^{2} d t, \quad i=1, \cdots, n$.

It can be proved $([10])$ that if one ignores the presence of other aircraft, the $J$-minimal maneuver for aircraft $i$ is the constant speed motion along the line segment joining $a_{i}$ to $b_{i}$. This serves perfectly for our purpose since this cost function favors maneuver with less travel distance and less speed variance at the same time.

Finally, we can formulate the constrained optimization problem we shall deal with as follows:

$$
\text { Minimize } J(\alpha) \text { subject to } \alpha \in \mathbf{P}(R) \text {. }
$$

\subsection{Necessary Conditions for Optimality}

As a first step, we shall derive various necessary conditions for an $n$-maneuver $\alpha^{*}=\left(\alpha_{1}^{*}, \cdots, \alpha_{n}^{*}\right) \in \mathbf{P}(R)$ to be a solution to problem (1). The machinery employed here is that of variational analysis, namely, if $\alpha^{*}$ is optimal, a local perturbation along any direction will not decrease the cost. A major obstacle to the application of the classic calculus of variations technique to this problem is the presence of the MSE constraint. One has to consider only perturbations that 


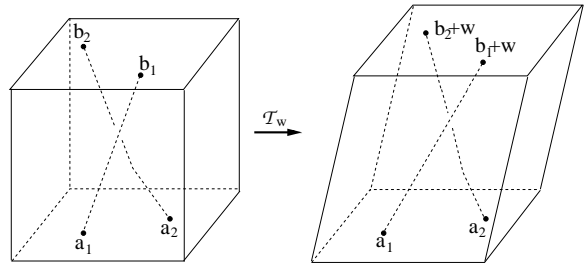

Figure 2: Tilt operation $\mathcal{T}_{w}$ on braids.

will lead to resolution maneuvers, i.e., to maneuvers in $\mathbf{P}(R)$, which is not guaranteed in general. It turns out that the braid representation of maneuvers introduced in Section 2 is useful in this respect, since it makes quite intuitive some transformations of resolution maneuvers which leave the MSE invariant. These transformations can then be used to generate legitimate perturbations to $\alpha^{*}$ and derive necessary conditions for optimality.

Let us introduce some notations for convenience. Given the set of starting and destination positions of $n$ aircraft $\mathbf{a}=\left(a_{1}, \cdots, a_{n}\right)$ and $\mathbf{b}=\left(b_{1}, \cdots, b_{n}\right)$, we use $\mathbf{P}(R, \mathbf{a}, \mathbf{b})$ and $\mathbf{P}(\mathbf{a}, \mathbf{b})$ to highlight the dependence of $\mathbf{P}(R)$ and $\mathbf{P}$ on $\mathbf{a}, \mathbf{b}$. This is useful when there is more than one set of starting or destination positions. A transformation on $\mathbf{P B}(\mathbf{a}, \mathbf{b})$ preserving the MSE is the tilt operation. Let $w \in \mathbf{R}^{2}$ be an arbitrary vector. Denote by $\mathbf{b}+w$ the $n$-tuple $\left(b_{1}+w, \cdots, b_{n}+w\right)$.

Definition 4 (Tilt Operator $\mathcal{T}_{w}$ ) The tilt operator $\mathcal{T}_{w}: \mathbf{P}(R, \mathbf{a}, \mathbf{b}) \rightarrow \mathbf{P}(R, \mathbf{a}, \mathbf{b}+w)$ maps each $\alpha \in$ $\mathbf{P}(R, \mathbf{a}, \mathbf{b})$ to $\beta=\mathcal{T}_{w}(\alpha) \in \mathbf{P}(R, \mathbf{a}, \mathbf{b}+w)$ such that

$$
\beta_{i}(t)=\alpha_{i}(t)+\frac{t-t_{0}}{t_{f}-t_{0}} w, \quad t \in T_{h}, \quad i=1, \cdots, n .
$$

Note that this definition is consistent since $\beta$ indeed satisfies $\Delta(\beta) \geq R$ (actually $\left\|\alpha_{i}(t)-\alpha_{j}(t)\right\|=\| \beta_{i}(t)-$ $\beta_{j}(t) \|$ for all $i, j$ and $\left.t\right)$. Figure 2 plots the tilt operation on the braid representation of some 2-maneuver.

It is easily checked that $\mathcal{T}_{w}$ is a bijection. Furthermore,

Proposition 2 Suppose $\alpha^{*}$ is an optimal solution to problem (1) in $\mathbf{P}(R, \mathbf{a}, \mathbf{b})$. Then, $\beta^{*}=\mathcal{T}_{w}\left(\alpha^{*}\right)$ is an optimal solution to problem (1) in $\mathbf{P}(R, \mathbf{a}, \mathbf{b}+w)$.

Proof: $\quad$ For any $\alpha \in \mathbf{P}(R, \mathbf{a}, \mathbf{b})$ let $\beta=\mathcal{T}_{w}(\alpha)$, then

$$
J(\beta)-J(\alpha)=\frac{w^{T}\left(2 \sum_{i=1}^{n} b_{i}-2 \sum_{i=1}^{n} a_{i}+n w\right)}{t_{f}-t_{0}},
$$

which is a constant independent of $\alpha$.

Consider arbitrary starting and destination positions a and $\mathbf{b}$, and set $\mathbf{b}^{\prime} \triangleq \mathbf{b}+w$ where $w=\frac{1}{n} \sum_{i=1}^{n}\left(a_{i}-b_{i}\right)$. Then, $\mathbf{a}$ and $\mathbf{b}^{\prime}$ are aligned in the sense that they have the same centroid, i.e., $\frac{1}{n} \sum_{i=1}^{n} a_{i}=\frac{1}{n} \sum_{i=1}^{n} b_{i}^{\prime}$. By Proposition 2, solving problem (1) for aligned $\mathbf{a}$ and $\mathbf{b}^{\prime}$ is equivalent to solving (1) for general $\mathbf{a}$ and $\mathbf{b}$.
The next operation we introduce is the drift operation. Let $\gamma: T_{h} \rightarrow \mathbf{R}^{2}$ be a continuous and piecewise smooth map such that $\gamma\left(t_{0}\right)=\gamma\left(t_{f}\right)=0$.

Definition 5 (Drift Operator $\mathcal{D}_{\gamma}$ ) The drift operator $\mathcal{D}_{\gamma}: \mathbf{P}(R, \mathbf{a}, \mathbf{b}) \rightarrow \mathbf{P}(R, \mathbf{a}, \mathbf{b})$ is a map such that for any $\alpha \in \mathbf{P}(R, \mathbf{a}, \mathbf{b}), \beta=\mathcal{D}_{\gamma}(\alpha) \in \mathbf{P}(R, \mathbf{a}, \mathbf{b})$ satisfies

$$
\beta_{i}(t)=\alpha_{i}(t)+\gamma(t), \quad t \in T_{h}, \quad i=1, \cdots, n .
$$

It is easily seen that $\mathcal{D}_{\gamma}$ is a bijection. In the braid representation, braid $\hat{\beta}$ is obtained by drifting braid $\hat{\alpha}$ according to $\gamma(t)$.

Assume that $\mathbf{a}, \mathbf{b}$ are aligned and $\alpha^{*}$ is the optimal solution to problem (1) in $\mathbf{P}(R, \mathbf{a}, \mathbf{b})$. For each $\lambda \in \mathbf{R}$, define $\beta(\lambda) \triangleq \mathcal{D}_{\lambda \gamma}\left(\alpha^{*}\right)$. Then $\beta(0)=\alpha^{*}$ and

$$
\begin{aligned}
\Delta J & \triangleq J(\beta(\lambda))-J\left(\alpha^{*}\right) \\
& =n \lambda^{2} \int_{t_{0}}^{t_{f}}\|\dot{\gamma}(t)\|^{2} d t+2 \lambda \int_{t_{0}}^{t_{f}} \dot{\gamma}(t)^{T} \sum_{i=1}^{n} \dot{\alpha}_{i}^{*}(t) d t .
\end{aligned}
$$

Since $\alpha^{*}$ is optimal, $\Delta J$ is nonnegative for all $\lambda$. Hence $\int_{t_{0}}^{t_{f}} \dot{\gamma}(t)^{T} \sum_{i=1}^{n} \dot{\alpha}_{i}^{*}(t) d t=0$. But this should hold for any choice of $\gamma$ such that $\gamma\left(t_{0}\right)=\gamma\left(t_{f}\right)=0$, say, $\gamma(t)=$ $\sum_{i=1}^{n} \alpha_{i}^{*}(t)-\sum_{i=1}^{n} a_{i}$, Therefore $\sum_{i=1}^{n} \dot{\alpha}_{i}^{*}(t)=0$ for almost all $t \in T_{h}$. Integrating, we get

Proposition $\mathbf{3}$ Assume that $\mathbf{a}, \mathbf{b}$ are aligned and $\alpha^{*}$ is an optimal solution to problem (1) in $\mathbf{P}(R, \mathbf{a}, \mathbf{b})$. Then

$$
\frac{1}{n} \sum_{i=1}^{n} \alpha_{i}^{*}(t)=\frac{1}{n} \sum_{i=1}^{n} a_{i}=\frac{1}{n} \sum_{i=1}^{n} b_{i}, \quad \forall t \in T_{h} .
$$

This optimality condition is sufficient to derive the analytical expression of the optimal maneuver for two aircraft encounters situations. Assume $\mathbf{a}=\left(a_{1}, a_{2}\right)$ and $\mathbf{b}=\left(b_{1}, b_{2}\right)$ are aligned, i.e., $\left(a_{1}+a_{2}\right) / 2=\left(b_{1}+b_{2}\right) / 2=$ $c$, for some $c \in \mathbf{R}^{2}$. If $\alpha^{*}=\left(\alpha_{1}^{*}, \alpha_{2}^{*}\right)$ is an optimal solution to problem (1), then by Proposition 3 , $\left[\alpha_{1}^{*}(t)+\alpha_{2}^{*}(t)\right] / 2 \equiv c$ throughout $T_{h}$. So to find $\alpha^{*}$, we need only to focus on those elements $\alpha \in \mathbf{P}$ satisfying $\left[\alpha_{1}(t)+\alpha_{2}(t)\right] / 2 \equiv c, t \in T_{h}$, and the MSE constraint becomes $\inf _{t \in T_{h}}\left\|\alpha_{1}^{*}(t)-c\right\| \geq R / 2$. For such maneuvers $J\left(\alpha_{1}\right)=J\left(\alpha_{2}\right)$, since $\dot{\alpha}_{1}(t)=-\dot{\alpha}_{2}(t)$ for all $t \in T_{h}$, and therefore problem (1) translates into

Minimize $J\left(\alpha_{1}\right)$, with $\alpha_{1} \in \mathbf{P}_{1}, \alpha_{1}: T_{h} \rightarrow B(c, R / 2)^{c}$,

where $B(c, R / 2)^{c}$ denotes the complement in $\mathbf{R}^{2}$ of the open disk $B(c, R / 2)$ of radius $R / 2$ centered in $c$.

Figure 3 shows one such $\alpha_{1}^{*}$. Basically, its image should be of minimal length, i.e., consist of one arc of $\partial B(c, R / 2)$ and two line segments $a_{1} p_{1}$ and $b_{1} q_{1}$ tangential to it. $p_{1}, q_{1}$ must be on the correct side of $\partial B(c, R / 2)$. Furthermore, to minimize energy, $\alpha_{1}^{*}$ must be of constant speed. In summary, 


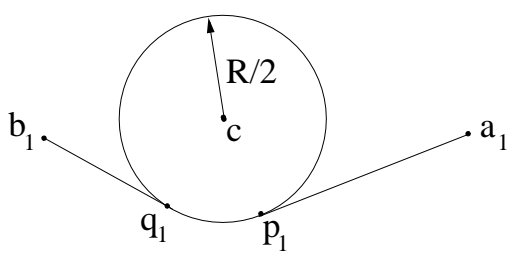

Figure 3: Construction of $\alpha_{1}^{*}$
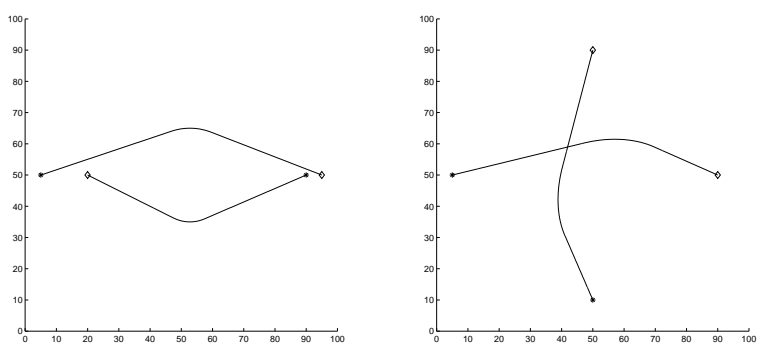

Figure 4: $\alpha^{*}$ for two aircraft encounters.

Lemma 1 The optimal solution to problem (2) is a constant speed motion consisting of three stages: first from $a_{1}$ to $p_{1}$ along a straight line, then from $p_{1}$ to $q_{1}$ along the circle $\partial B(c, R / 2)$, and last from $q_{1}$ to $b_{1}$ along a straight line.

Denote with $\gamma^{*}(\mathbf{a}, \mathbf{b})$ the global optimal solution to problem (2) given in Lemma 1, highlighting its dependence on the aligned points a, b. Using Proposition 2, we can now characterize the optimal maneuver for the case when $\mathbf{a}, \mathbf{b}$ are not necessarily aligned.

Theorem 2 In the case of a two aircraft encounter, the optimal solution $\alpha^{*}$ to problem (1) in $\mathbf{P}(R, \mathbf{a}, \mathbf{b})$ is:

$$
\left\{\begin{array}{l}
\alpha_{1}^{*}(t)=\gamma^{*}(\mathbf{a}, \mathbf{b}+w)(t)-\frac{t-t_{0}}{t_{f}-t_{0}} w, \\
\alpha_{2}^{*}(t)=a_{1}+a_{2}-\gamma^{*}(\mathbf{a}, \mathbf{b}+w)(t)-\frac{t-t_{0}}{t_{f}-t_{0}} w,
\end{array}\right.
$$

for all $t \in T_{h}$, where $w \triangleq\left(a_{1}+a_{2}-b_{1}-b_{2}\right) / 2$.

Figure 4 shows the plots of optimal resolution maneuvers for two typical two-aircraft encounters. Although in $(3), \gamma^{*}(\mathbf{a}, \mathbf{b}+w)$ is a constant speed motion, in general $\alpha_{1}^{*}$ and $\alpha_{2}^{*}$ are not, since they have an additional drift term: the larger the difference $w$ between $a_{1}+a_{2}$ and $b_{1}+b_{2}$, the larger the variation in speed.

By using other transformations of maneuvers which preserve the MSE, we can obtain additional optimality conditions. See [1] for details.

\subsection{Optimal 2-legged Maneuvers}

Due to the difficulty in computing the optimal joint resolution maneuver $\alpha^{*}$ for the general multiple aircraft resolution problem (1), we deal with the case of 2-legged maneuvers. Consider $n$ aircraft with starting positions $\mathbf{a}=\left(a_{1}, \cdots, a_{n}\right)$ and destination positions

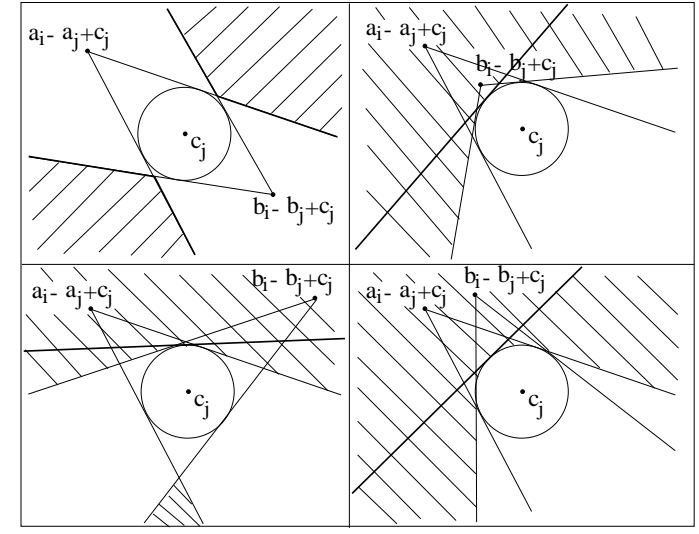

Figure 5: Feasible set for $c_{i}$ given $c_{j}$.

$\mathbf{b}=\left(b_{1}, \cdots, b_{n}\right)$. Fix an epoch $t_{1} \in\left(t_{0}, t_{f}\right)$ and associate with each aircraft $i$ a waypoint $c_{i}$. Then a 2-legged maneuver for aircraft $i$ is a constant speed motion consisting of 2 stages: first from $a_{i}$ at time $t_{0}$ to $c_{i}$ at time $t_{1}$ and then from $c_{i}$ at time $t_{1}$ to $b_{i}$ at time $t_{f}$, both along a straight line. Denote with $\mathbf{P}_{i}^{2}$ the set of all 2legged maneuvers for aircraft $i$, and with $\mathbf{P}^{2} \triangleq \prod_{i=1}^{n} \mathbf{P}_{i}^{2}$ the set of all 2-legged joint maneuvers. $\mathbf{P}^{2}(R)$ denotes the set of all elements of $\mathbf{P}^{2}$ with MSE at least $R$.

In this subsection, we shall try to solve the 2-legged version of problem (1) defined as follows:

$$
\text { Minimize } J(\alpha) \text { subject to } \alpha \in \mathbf{P}^{2}(R) \text {. }
$$

Compared with problem (1), problem (4) is finite dimensional, hence it can be solved by various standard optimization techniques. Notice that for $\alpha \in \mathbf{P}^{2}$ with waypoints $\left\{c_{i}\right\}_{i=1}^{n}$,

$$
J(\alpha)=\frac{t_{f}-t_{0}}{\left(t_{f}-t_{c}\right)\left(t_{c}-t_{0}\right)} \sum_{i=1}^{n}\left\|c_{i}-c_{i}^{u}\right\|^{2}+C
$$

where $C$ is a constant and $c_{i}^{u}$ is given by

$$
c_{i}^{u}=\frac{\left(t_{f}-t_{c}\right) a_{i}+\left(t_{c}-t_{0}\right) b_{i}}{t_{f}-t_{0}}, \quad i=1, \cdots, n .
$$

The MSE constraint can be simplified as well. For each aircraft pair $i$ and $j$, suppose we fix $c_{j}$ and have the freedom to choose $c_{i}$. Figure 5 shows the feasible set of $c_{i}$ by a shaded region, which has four possible configurations depending on the relative positions $a_{i}-a_{j}$ and $b_{i}-b_{j}$. In some configurations the feasible set has two connected components, corresponding to the two different resolution types for aircraft $i$ and $j$.

Suppose we have decided which type of resolution maneuver to use. Then, the problem is to find the waypoints $c_{1}, \cdots, c_{n}$ which

Minimize $\sum_{i=1}^{n}\left\|c_{i}-c_{i}^{u}\right\|^{2}$ subject to $c_{i} \in A_{i j}^{ \pm}\left(c_{j}\right), \forall i \neq j$, 

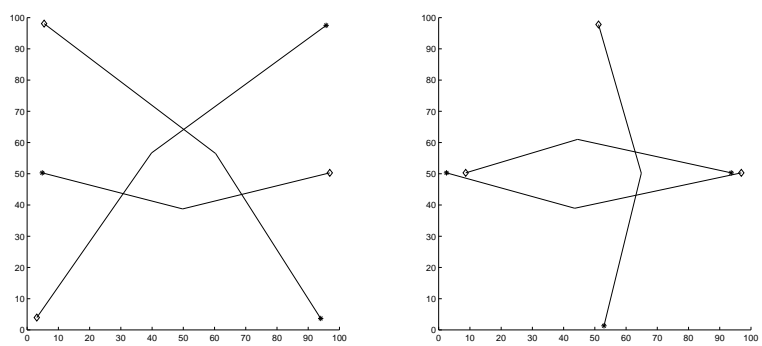

Figure 6: Optimal two legged resolution maneuvers for 3-aircraft encounters.

where $A_{i j}\left(c_{j}\right)$ denotes the feasible set of $c_{i}$ given $c_{j}$, and \pm denotes the connected component of $A_{i j}\left(c_{j}\right)$ matching our desired resolution type. Notice that in all but the first configuration, one of the connected component of $A_{i j}\left(c_{j}\right)$ is nonconvex. We then linearize the feasible set by using a half plane inner approximation $A_{i j}^{\prime}\left(c_{j}\right)$ of $A_{i j}\left(c_{j}\right)$ as shown in Figure 5 . In the special case when any pair of aircraft is in the first configuration, which corresponds to the case when the unconstrained optimal joint maneuver would cause a conflict between any aircraft pair, the approximation is tight.

We then have a linearly constrained quadratic optimization problem which can be solved very efficiently by many software packages. Simulation results for 3aircraft encounters are shown in Figure 6. In both cases, each pair of the three aircraft is in the first configuration. The optimal maneuver is calculated for each type and then the global optimal one is selected.

\section{Randomized Algorithm}

In [4], we propose a decentralized conflict resolution algorithm for multiple aircraft encounters based on a probabilistic model of the aircraft motion. Although this stochastic algorithm performs reasonably well, one of its drawbacks is that safety cannot be guaranteed. On the other hand, the optimization algorithm we proposed in the previous section ensures safety, but it cannot handle the explosively increasing number of resolution types when the number of aircraft involved is large. We then suggest to use the stochastic resolution algorithm as the random "type chooser" for the optimization procedure, thus obtaining a randomized optimization procedure which both guarantees the safety property and is computationally efficient.

More specifically, for a given encounter of multiple aircraft, we run the stochastic resolution algorithm first. The generated maneuver corresponds to a particular resolution type which can be thought to be a relatively good one. Using this type we can then run the convex optimization algorithm to obtain a nearly optimal resolution maneuver within that type.

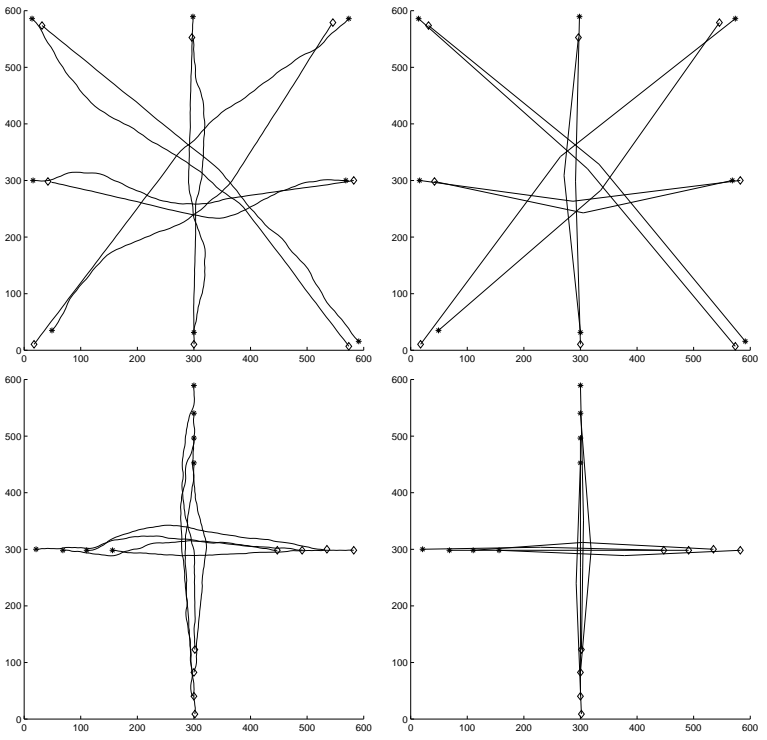

Figure 7: Resolution maneuvers for 8-aircraft encounters.

Simulation results of the randomized algorithm for two 8-aircraft encounters are shown in Figure 7, where the left column shows the simulation results of the stochastic algorithm and the right column shows the results of the convex optimization process for the particular type of maneuvers specified by the left column.

\section{References}

[1] J. Hu, "A study of conflict detection and resolution in free flight," M.S. thesis, UC, Berkeley, 1999.

[2] M.S. Nolan, Fundamentals of Air Traffic Control, Brooks/Cole, Wadsworth, 3rd edition, 1998.

[3] G.J. Pappas, C. Tomlin, J. Lygeros, D. Godbole, and S. Sastry, "A next generation architecture for air traffic management systems," in IEEE CDC, San Diego, CA, 1997.

[4] J. Hu, J. Lygeros, M. Prandini, and S. Sastry, "Aircraft conflict prediction and resolution using Brownian Motion," in IEEE CDC, Pheonix, AZ, 1999.

[5] J. Kosecka, C. Tomlin, G. Pappas, and S. Sastry, "Generation of Conflict Resolution Maneuvers For Air Traffic Management," in IEEE Conf. on Intelligent Robotics and System '97, 1997.

[6] N. Durand, J.M. Alliot, and J. Noailles, "Automatic aircraft conflict resolution using genetic algorithms," in 11th ACM Conf. on Applied Comp., Philadelphia, 1996.

[7] E. Frazzoli, Z.-H. Mao, J.-H. Oh, and E. Feron, "Resolution of conflicts involving many aircraft via semidefinite programming," AIAA Journal of Guidance, Control and Dynamics, to appear.

[8] J. Kuchar and L.C. Yang, "Survey of conflict detection and resolution modeling methods," in AIAA Guidance, Navigation, and Control Conf., New Orleans, LA, 1997.

[9] S. Morgan, The mathematical theory of knots and braids: an introduction, North-Holland, 1991.

[10] J.W. Milnor, Morse theory, Annals of mathematics studies, 51. Princeton University Press, 1963, based on lecture notes by M. Spivak and R. Wells. 